\title{
Applicability Assessment of UAV Mapping for Disaster Damage Investigation in Korea
}

\author{
S. S. Kim ${ }^{1}$, T. H. Kim ${ }^{1}$, J. S. Sim ${ }^{1}$ \\ ${ }^{1}$ National Disaster Management research Institute, 365 Jongga-ro, Jung-gu, Ulsan, 44538, Rep. of Korea - (sskim73, ddahoon,
} js2309)@korea.kr

Commission VI, WG VI/4

KEY WORDS: UAVs, Rapid Drone Mapping, Natural Disaster, Damage Investigation

\begin{abstract}
:
As natural disaster occurs, the local and the central government should investigate the damaged fields promptly, analyze quantitatively the degree of damage, and establish an appropriate disaster recovery plan in accordance with Framework Act on the Management of Disasters and Safety in Korea. The purpose of this study is to assess the applicability of UAV photogrammetry for the management of natural disaster. First, we suggest small easy-to-use UAV-based investigation procedure for natural disaster damaged area in the phase of disaster recovery in Korea. Before drone-based aerial surveying, the field survey can be performed with DGPS RTK for GCPs setting-up around disaster site. In this paper, we generate three dimensional terrain information and highresolution ortho-imagery and then analyse quantitatively damage degree by natural disaster using commercial UAVs and drone mapping technique. Finally, we evaluate the mapping accuracy and work efficiency of drone mapping for disaster investigation application through comparing with traditional investigation work process which was dependent on labour-intensive field survey. The resolution ortho-image map of within less $5 \mathrm{~cm}$ of GSD generated by aerial photographs acquired from UAVs at the altitude of $100 \mathrm{~m} 250 \mathrm{~m}$ enabled us to check damage information such as facilities destroy or the trace of soil erosion around the river flooded and reservoir collapsed area. In addition, three dimensional point cloud data of landslide-damaged areas enabled us to more accurately measure the width and the depth of outflows caused by landslides, soil runoff distance, and landslide damage area. The photogrammetry-based drone mapping technology for the disaster damage investigation is expected to be an alternative approach to support or replace the labour-intensive disaster site survey that needs to investigate the disaster site quickly and timely.
\end{abstract}

\section{INTRODUCTION}

Due to global climate changes and rapid urbanization, our society has been faced with difficult and unpredictable disasters. Natural disaster closely related to meteorological phenomena and geographic factors can reduce slightly damage extent by taking preparative and preventive measures. For effective and systematical disaster management, far-reaching researches and promising technical development have been carried out actively to minimize the damages by natural disaster using advanced observation platforms and observation sensors: satellite-based and aerial mapping platform, high-precision mobile mapping system (MMS), UAV-based LiDAR, etc.

Despite the dramatic growth of these technologies, socioeconomic losses and casualties caused by natural disaster have been increased in Korea due to rapid urbanization and global climate change. The total cost of recovery by natural disaster is estimated about 400 million US dollars per a year over past decades. Most of the damage by natural disaster around the Korean Peninsula has been caused by flood and landslide such as a typhoon, a heavy rainfall for the rainy season. Occurring natural disaster in Korea, the local and the central government should investigate the damaged sites promptly, analyse quantitatively the extent of damage, and establish an appropriate disaster recovery plan in accordance with Framework Act on the Management of Disasters and Safety.

Since 2013, Korea's National Disaster Management research Institute (NDMI), responsible for implementing R\&D related to national disaster and safety management, has studied for disaster scientific investigation (DSI). DSI, a highly organized framework to find the root cause of disasters, aims to implement, monitor and feedback with disaster profiling through state-ofthe-art forensic technologies. As the feasible operational tools for DSI, NDMI has started to adapt and operate the various types of investigation platforms and devices: a MMS-type specialized vehicle, UAVs, ultrasonography detector, rebar detector, etc. (Kim et al, 2018).

In recent years, Unmanned Aerial Vehicle (UAV) with various on-board sensors is considered to be a cost-effective tool for large scale aerial mapping. The suitability of small drones for mapping applications is dependent on mapping extent, geometric accuracy, the durability such as flight time and control distance, and the lifting capacity of UAV. It is equipped with the GNSS/IMU, MEMS, gyroscopes, accelerometers, and barometer to conduct direct sensor orientation. Precise timetagging of the camera shutter and GNSS time enable it to annotate the position and attitude data on the metadata of captured imagery (Rehak et al., 2013). As an alternative to AT (Aerial Triangulation), direct geo-referencing of airborne sensor is to measure the position and orientation of an airborne mapping sensor so that each pixel or range can be georeferenced to the specific map projection system without any kind of ground information collected in the field (Mian et al., 2015). Direct Geo-referencing is a suitable process to create of accurate map products rapidly from UAV aerial imagery with minimal GCPs or without GCPs. This is efficient to produce the newest map rapidly where does not allow to approach such as disaster area needed emergent response.

UAV mapping enable field investigator to generate the damaged map where does not allow to approach such as disaster area. The accuracy of on-board GNSS in UAV is however significantly lower than the accuracy of GPS used in general aerial surveying due to its own specification. Thus, related further researches are required to improve the accuracy of map.

This study aims to propose a photogrammetry-based drone mapping approach for the damage investigation by natural 
disaster and to assess its applicability for timely natural disaster mapping and monitoring.

\section{STUDY AREA AND METHOD}

\subsection{Area of Study}

The study sites were selected on considerable damaged areas where landslides, river floods, and reservoir collapses have occurred due to heavy rains and a typhoon in 2018 .

From late June to early July 2018 , there were heavy rainfalls around southern area of Korean peninsula. In this paper, the study sites were selected on considerable damaged areas where landslides, river floods, and reservoir collapses have occurred due to heavy rains and a typhoon at that time (Figure 1).

During this period, due to the heavy and continuous rainfall in Boseong-gun, more than $15 \mathrm{ha}$ of agricultural area was flooded and the landslides occurred in 17 steep mountainous areas in national and private forests. Cumulative precipitation during this period was $236 \mathrm{~mm} \sim 327.5 \mathrm{~mm}$ around Jellanam-do.

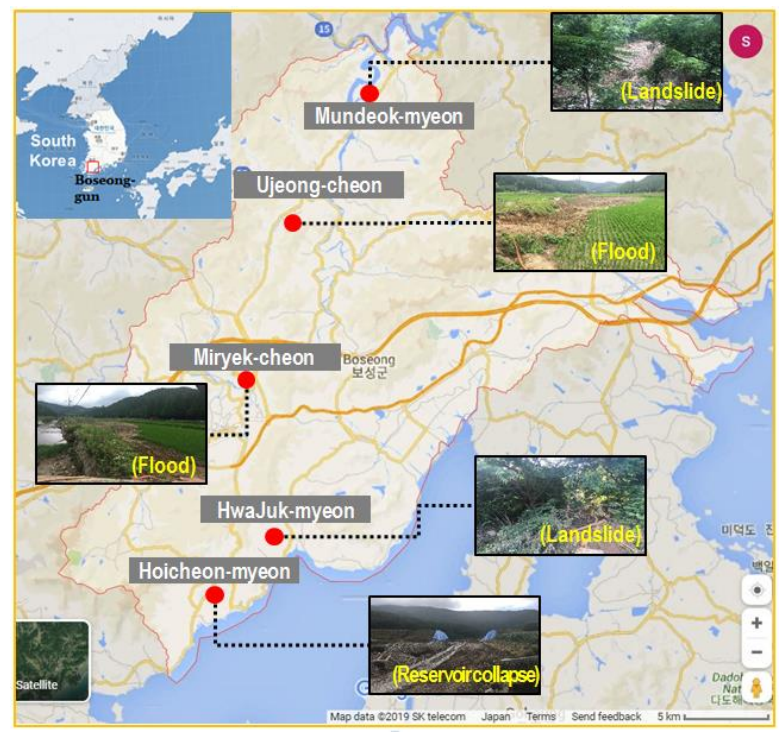

Figure. 1. Study area

\subsection{UAV System}

The small rotor wing and the fixed wing drones were adopted in the paper for the natural disaster site investigation.

Drones for investigating damages occurred by natural disaster were selected focusing on aspects of safety, performance, and usability.

DJI's Inspire2 is a commercial drone with a relatively stable flight performance and has a battery capacity of about 25 minutes with longer endurance flight than the Inspire1. Zenmuse X5S camera is mounted with a 3-axis gimbal which is suitable for taking aerial photography for drone mapping.

A fixed-wing FireFly6 being capable of a vertical take-off and landing, is a rotor-tilling type drone on a flight mission, so it does not need a plenty of runway space for take-off and landing, which is advantageous for disaster field operation. A fixed wing drone enable users to carry out mapping mission with relatively stable and efficient flight performance, so it has advantages for long flight mapping in wide area disaster area.

Sony A6000 optical camera mounted on FireFly $6^{\mathrm{TM}}$ has a builtin 24.3MP APS-C sensor that allows users to collect high- resolution aerial photographs with a $20 \mathrm{~mm}$ lens and a GSD of $2.36 \mathrm{~cm}$ at a flight altitude of $120 \mathrm{~m}$.

Recently, it is possible to acquire relatively precise flight information of the drone through a post-processing kinematic RTK (PPK) technology. In addition, a variety of multi-sensing data around disaster area can be collected from the multispectral sensor or the thermal camera depending on the purpose of applications.

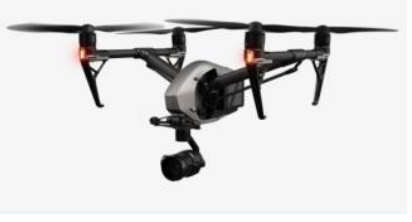

(a) Inspire2

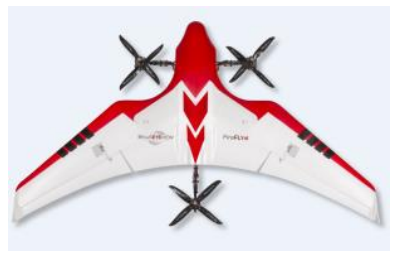

(b) FireFly6
Figure. 2. Small UAVs of this study

\begin{tabular}{|l|c|c|}
\hline & Inspire2 & FireFly6 \\
\hline Wing span $(\mathrm{mm})$ & 0.6 & 1.5 \\
\hline Wing type & 4 rotaries & Fixed wings \\
\hline Cruise speed (m/s) & $22 \sim 25$ & $15 \sim 18$ \\
\hline Max. endurance time (min.) & 25 & $50 \sim 60$ \\
\hline Flight altitude (m) & 150 & 150 \\
\hline Payload $(\mathrm{kg})$ & 0.7 & 0.7 \\
\hline Wind resistance (m/s) & $\leq 10$ & $\leq 7$ \\
\hline Launch/landing & Vertical & VTOL \\
\hline Flight range (km) & 3.5 & $5 \sim 53$ \\
\hline Mounted sensors & Zenmuse X5S & $\begin{array}{c}\text { Sony A6000, } \\
\text { MicaSense, } \\
\text { RedEdge }\end{array}$ \\
\hline
\end{tabular}

Table 1. Specifications of small UAVs used in this study

\begin{tabular}{|l|c|c|}
\hline & Sony A6000 & Zenmuse X5S \\
\hline Camera size $(\mathrm{mm})$ & $120 \times 67 \times 45$ & $140 \times 98 \times 132$ \\
\hline Sensor type $(\mathrm{mm})$ & $\begin{array}{c}\text { CMOS(23.5×15.6) } \\
, \text { APS-C }\end{array}$ & $4 / 3^{\prime \prime}$ CMOS \\
\hline Lens Focal length & $16 \sim 50 \mathrm{~mm}$ & $15 \mathrm{~mm}$ \\
\hline Field of View & $62^{\circ}$ & $72^{\circ}$ \\
\hline Max. shutter speed & $1 / 4,000 \mathrm{~s}$ & $8 \sim 1 / 8,000 \mathrm{~s}$ \\
\hline $\begin{array}{l}\text { Effective sensor } \\
\text { resolution }\end{array}$ & $24.3 \mathrm{MP}$ & $16 \mathrm{MP}$ \\
\hline $\begin{array}{l}\text { Still image } \\
\text { resolution }\end{array}$ & $6,000 \times 4,000$ & $4,608 \times 3,456$ \\
\hline Weight $(\mathrm{g})$ & $344 \mathrm{~g}$ & $115 \mathrm{~g}$ \\
\hline
\end{tabular}

Table 2. Specifications of camera mounted UAVs

\subsection{Data processing for Drone Mapping}

Unmanned Aerial Vehicles (UAV) with various types of onboard sensors are considered to be a cost-effective tool for large-scaled aerial mapping. Direct geo-referencing of UAV sensors measuring the position and orientation of a built-in camera enable to create map products in real-time and rapidly 
so that it is suitable to utilize for disaster response applications (Kim et al., 2019).

Drone mapping is a simplified aerial photogrammetry-based data processing procedure using ultra-small built-in MEMS of a drone, though the accuracy of the IOPs or the EOPs for the nonmetric camera mounted on the drones is somewhat poor.

Through the iterative bundle adjustment process using high capacity computing technology, drone mapping can generate the high-precise 3D terrain model and the ortho-image similar to the accuracy of the conventional aerial photogrammetry by optimization of IOPs and EOPs.

In this study, the process of drone mapping is as follows: 1) flight planning, 2) GNSS-RTK surveying, 3) aerial photos capturing, 4) keypoints extraction and image matching, 5) 3D point cloud extraction, 6) DSM/DEM generation, and finally 7) ortho-image generation. Before generating DSM and DEM, accurate $3 \mathrm{D}$ point clouds were generated by extracting points of interest, optimizing camera models optimization, and improving the accuracy of image matching. In order to conduct drone mapping, we used Pix4Dcapture and FireFlyplanner for flight planning and aerial photographs acquisition, and Pix4Dmapper ${ }^{\mathrm{TM}}$ and Agisoft PhotoScan for aerial image processing acquired by a drone.

\begin{tabular}{|c|}
\hline Mission planning : flight height / time / path / overlap etc. \\
\hline$\downarrow$ \\
\hline Data pre-process : system / camera calibration \\
\hline$\downarrow$ \\
\hline Drone aerial Imaging : manually / semi-auto. flight / capturing \\
\hline \\
\hline Measurement of tie points : point extraction / matching \\
\hline Measurement of control points : RTK-GNSS surveying \\
\hline Image orientation: IOPs/EOPs orientation/camera model optimization \\
\hline$\downarrow$ \\
\hline DTM/DSM generation : point clouds densification / filtering \\
\hline Ortho-image generation : automatic image mosaic, blending \\
\hline Map Products assessment : accuracy check \\
\hline
\end{tabular}

Figure 3. Procedure of drone mapping

\section{DRONE MAPPING FOR NATURLA INVESTIGATION}

Natural disaster damage investigation in Korea

In general, disaster management consists of following four steps: prevention, preparation, response, and recovery.

When natural disaster occurs in Korea, disaster damage investigation led by local government is carried out as follows; disaster damage reporting, field investigation, damage analysis, recovery planning and implementation.

According to the Disaster Recovery Guideline under Framework Act on the Management of Disasters and Safety, the heads of the local government in damaged area by natural disaster should promptly the disaster site within the legal period (about 10 days) and report damage status for facilities to the central government through National Disaster Management System (NDMS), and then establish a recovery plan and implement it.

If there is more damage than the recovery cost support criteria of the central government determined by the financial index of the local government, the central disaster safety headquarters will investigate directly and then judge whether or not to declare special disaster area. (MOIS, 2018).
Local government should register the damage states of facilities by natural disaster on the disaster register of the National Disaster Management System (NDMS), and all procedures in the disaster recovery phase for the disaster management such as the damage investigation of the disaster site, the damaged level analysis, the recovery cost calculation, and recovery implementation, etc. are managed through NDMS.

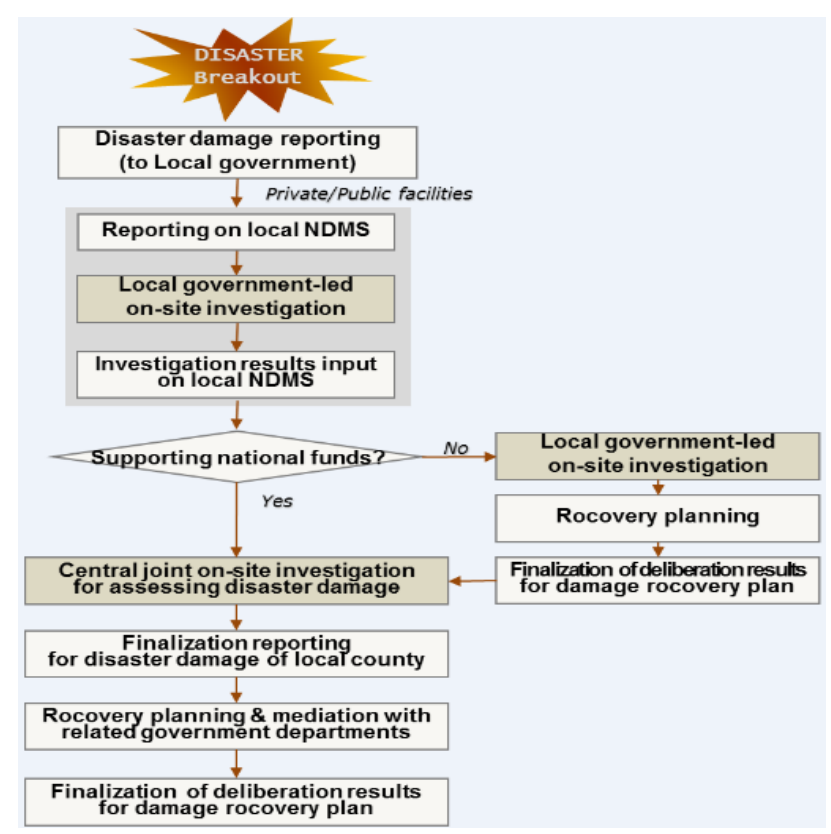

Figure 4. Disaster investigation procedures in Korea

\subsection{Aerial photo capturing on Disaster site}

In this study, the rotary and the fixed wing drones were utilized for damage investigation at five sites: 2 landslides, 2 floods, and 1 collapse of the reservoir, respectively.

Aerial photographs of 5 damaged areas were collected from on-board optic camera of drones and post-processed by drone mapping procedure to assess the quantitative damage level.

We planned to capturing aerial imagery at flight heights of $100 \sim 150 \mathrm{~m}$. A total of 1110 images were acquired at 5 damaged sites: 264 images at 2 landslide site, 579 images at 2 flooded area, 267 reservoir collapse site, respectively.

The aerial images collected from drones are as follows (Table 3).

\begin{tabular}{|c|c|c|c|c|}
\hline $\begin{array}{c}\text { Acquisition } \\
\text { time }\end{array}$ & $\begin{array}{c}\text { Damaged } \\
\text { sites }\end{array}$ & $\begin{array}{c}\text { Disaster } \\
\text { type }\end{array}$ & $\begin{array}{c}\text { Collected } \\
\text { imagery }\end{array}$ & $\begin{array}{c}\text { UAVs } \\
\text { type }\end{array}$ \\
\hline 11 July, 2018 & Mundeok & Landslide & 123 & Rotary \\
\hline \multirow{3}{*}{ 12 July,2018 } & Hoichen & $\begin{array}{c}\text { Reservoir } \\
\text { collapse }\end{array}$ & 267 & $\begin{array}{c}\text { Fixed wings/ } \\
\text { Rotary }\end{array}$ \\
\cline { 2 - 5 } & Hwajuk & Landslide & 141 & Rotary \\
\cline { 2 - 5 } & $\begin{array}{c}\text { Miryek river } \\
\text { FJeong } \\
\text { river }\end{array}$ & Flooding & 251 & Rotary \\
\hline
\end{tabular}

Table 3. Aerial imagery of damaged areas acquired from UAVs 


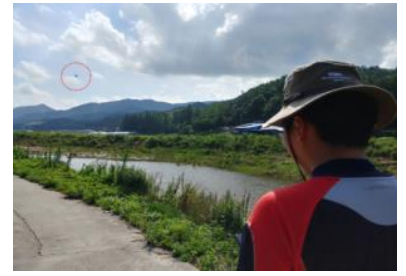

(a) Imaging by Inspire2

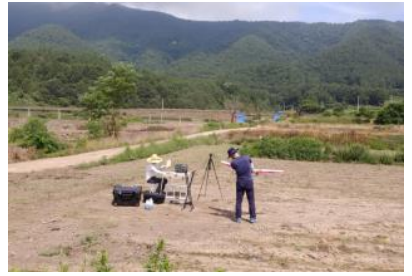

(b) Pre-test of FireFly6
Figure. 5. Small UAVs operation in disaster field

\subsection{Natural disaster drone mapping}

In fact, considering the limitation of work to be done in the hard-to-reach disaster area where the damage investigation requiring time-consuming works such as the field photographing and the damage surveying depended on tapeline or eye measuring is not easy to conduct within a short investigation period (about 10 days). Therefore, it needs to apply the current technology level of drone mapping capable of quickly capturing and mapping aerial photographs at inaccessible disaster sites.

Direct Geo-referencing is a suitable process to create of accurate map products rapidly from UAV aerial imagery with minimal GCPs. This technology is efficient to produce the newest map rapidly where does not allow to access such as disaster area. Direct geo-referencing in UAV photogrammetry is to measure the position and orientation of an on-board camera directly so that each pixel can be geo-referenced to the Earth without GCPs. It requires precise location and attitude information for the on-board camera mounted on the gimbal of UAV. The accuracy of the GNSS/INS mounted on the UAV, however, is not enough to utilize the information as it provides. A complementary task is necessary for improving the accuracy (Kim et al., 2019).

The accuracy of the mapping using the small drones depends on various factors such as the terrain of the mapping area, the precision of the on-board sensors of drone, the image overlap/sidelap rate, weather conditions, flight speed and stability of drone, GNSS surveying conditions, etc.

In the case of mapping using geo-tagged image information obtained in autonomous flight mode from barometer and GNSS /INS mounted on the drone without GCPs, the previous related literature says the accuracy of drone mapping using small drones is expected about 1 2 times of GSD horizontally and 1 3 times of GSD vertically for a correct estimation model (Pix4D, 2019, Küng et al., 2011). In addition, drone mapping using GCPs can improve the geo-referencing accuracy with $\mathrm{cm}$ level, although there are some differences depending on the distribution and the number of GCPs and the accuracy of GNSS surveying.

In this study, the horizontal accuracy of the result generated by performing drone mapping without GCPs in a dangerous and difficult-to-reach disaster sites. In the case of drone mapping of a mountainous region with an irregular altitude variance at the flight altitude of $200 \mathrm{~m}$, the maximum horizontal position error was about $9 \mathrm{~m}$. In the flat area, the maximum error is measured with $1 \sim 2.3 \mathrm{~m}$.

The damage analysis by natural disaster is carried out using 3D map data (Point Cloud, DSM/DEM), Ortho-imagery based on location-based GIS data derived by using Pix4D Mapper and Agisoft PhotoScan.

\begin{tabular}{|c|c|c|c|}
\hline Damaged sites & $\begin{array}{c}\text { Type of } \\
\text { disaster }\end{array}$ & $\begin{array}{c}\text { RMS X } \\
{[\mathrm{m}]}\end{array}$ & $\begin{array}{c}\text { RMS Y } \\
{[\mathrm{m}]}\end{array}$ \\
\hline Mundeok & Landslide & 2.3 & 8.8 \\
\hline Hoichen & $\begin{array}{c}\text { Reservoir } \\
\text { collapse }\end{array}$ & 1.1 & 3.2 \\
\hline Miryek & Flooding & 2.2 & 3.5 \\
\hline
\end{tabular}

Table 4. Geo-corrction accuracy small drone mapping

\subsection{Natural disaster damage analysis}

\subsubsection{Damage analysis by landslide}

In the Moonduk area where road facilities has been lost due to landslides and soil erosion, 89 drone photographs were taken and the ortho-image map of about $3 \mathrm{~cm}$ level with spatial resolution was finally generated through drone mapping.

The total drone aerial mapping area was about $89,497.29 \mathrm{~m}^{2}$, the landslide damage area on the image map was about 3,333.5 $\mathrm{m}^{2}$ and the whole runoff length by the landslide was about $332.9 \mathrm{~m}$. The mountainous area occurred a landslide can be restrictions on the drone mapping because it is not only difficult to operate with the line-of-sight flight of drones but also generate accurate 3D terrain model due to occlusion area under the dense and thick forest. It is also expected that the landslide investigation of mountainous terrain which is difficult to guarantee the quality of drone mapping due to irregular altitudes can qualitatively investigate the overall damage situation and environmental landslide inducing factors in the inaccessible damage area by using videos sequences captured drones.

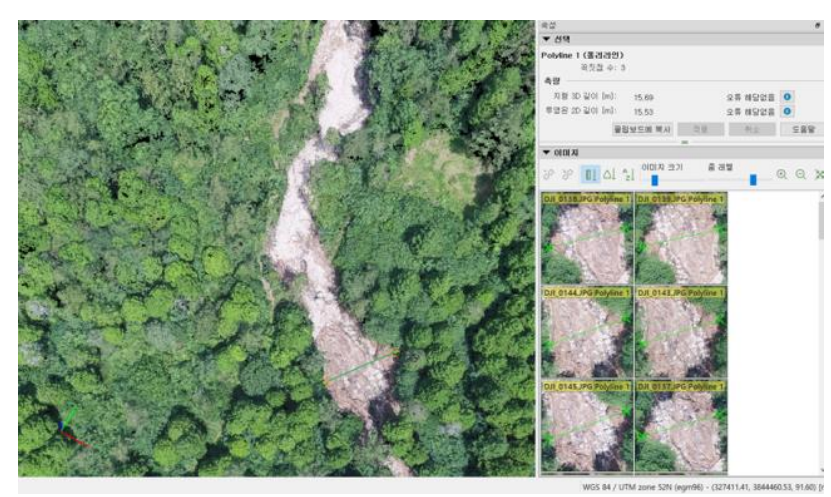

Figure. 6. Landslide damage analysis using drone mapping

\subsubsection{Damage analysis by reservoir collapse}

The total 267 drones aerial photographs were taken into the area where debris outflow occurred as a result of the collapse of reservoir due to strong heavy rain, and an ortho-image map of about $4 \mathrm{~cm}$ level with spatial resolution was generated.

The total drone aerial mapping area was is about $1.5 \mathrm{~km}^{2}$, and the soil leak area of the river bank on the generated 3D terrain model and ortho-image map is about $1.3 \mathrm{~km}$, and the damaged area estimated based on the runoff trace on the ortho-image map is about $24,248.8 \mathrm{~m}^{2}$.

The decay length between the top of the reservoir was about $23.9 \mathrm{~m}$ and the whole bank distance was about $129 \mathrm{~m}$ on the $3 \mathrm{D}$ terrain model. As the damaged area due to disasters is wide and mapping coverage increases, the fixed wing with the longer 
flight endurance is expected to be more efficient the rotor wing drones.

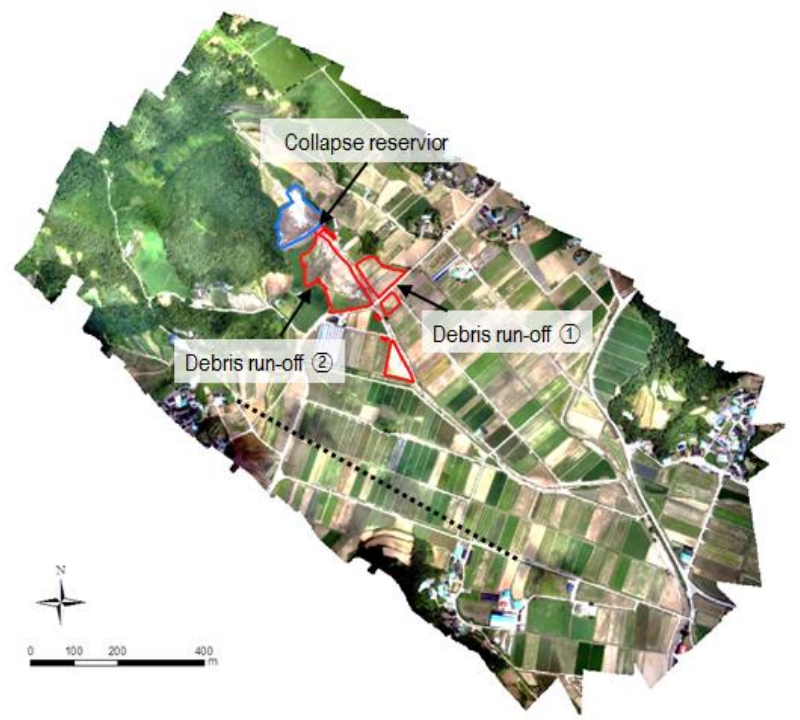

Figure. 7. Flood damage analysis by reservoir collapse

\subsubsection{Damage analysis by flood}

Many river facilities were lost during long heavy rainfall and floods and debris occurred at this time was run off into agricultural area. For investigate these areas, a total of 249 aerial photos were taken and produced 3D DSM/DEM and an ortho-image map with a spatial resolution of $2 \mathrm{~cm}$ level.

The drone mapping area was about $90,778.1 \mathrm{~m}^{2}$ and debris runoff area into the agricultural land on the mapping area was analysed to be about $4,390.6 \mathrm{~m}^{2}$.

Inundation and loss of facilities occurred mainly in agricultural area and around river facilities such as bridges, river weirs, bank protection facilities, access road along the bank of river, etc. The quantitative flood damage analysis results; flooded area in farmland, levee leakage, and collapse distance etc. can be utilized as objective investigation data for establishing natural disaster recovery plan.

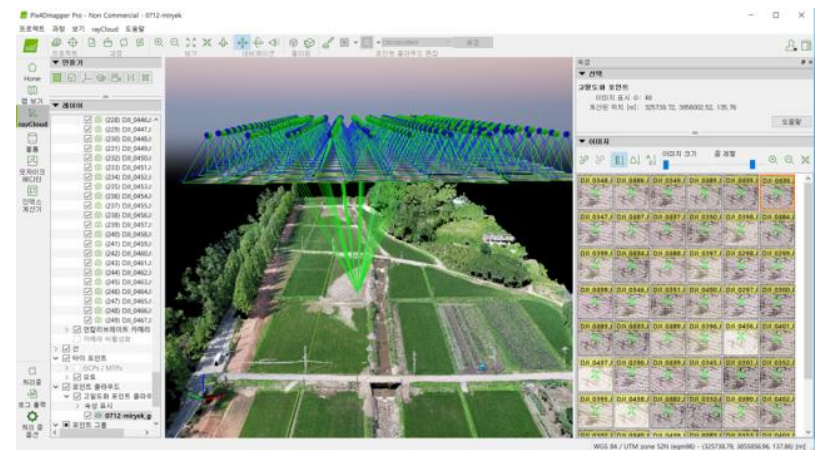

Figure. 8. Flood damage analysis using drone mapping

\section{CONCLUSIONS}

Natural disasters, which are closely related to global weather, climate, and environmental factors, can be effective in reducing damage by proactive preparation and prevention compared to social hazards that are difficult to predict. Therefore, the promising technologies development and far-reaching research for recent UAVs related to disaster management are actively in progress to minimize damage extent through timely and effective preparation, prevention, response, recovery of disasters.

In this paper, we suggest an investigation approach of disaster damage using commercial small drones for five disaster sites such as in-situ landslide, river flooding, and reservoir collapse areas through photogrammetry-base drone mapping at the flight altitude of 100 200m, and perform the quantitative analysis of natural disaster damage extent, finally we can get following conclusions;

First, we generate 3D terrain information and high-resolution ortho-imagery and then analyse quantitatively damage degree by natural disaster using commercial UAVs and drone mapping technique. We also evaluate the mapping accuracy and work efficiency of drone mapping for disaster investigation application through comparing with traditional investigation work process which was dependent on labour-intensive field survey. The maximum horizontal position error was about $9 \mathrm{~m}$ at irregular height mountainous area, but, in the flat area, the maximum error is measured with $1 \sim 2.3 \mathrm{~m}$.

Second, the resolution ortho-image map of within less $5 \mathrm{~cm}$ of GSD generated by aerial photographs acquired from UAVs at the altitude of $100 \mathrm{~m} \sim 250 \mathrm{~m}$ enabled us to check damage information such as facilities destroy or the trace of soil erosion around the river flooded and reservoir collapsed area.

Finally, three dimensional point cloud data of landslidedamaged areas enabled us to more accurately measure the width and the depth of outflows caused by landslides, soil runoff distance, and landslide damage area.

The photogrammetry-based drone mapping technology for the disaster damage investigation is expected to be an alternative approach to support or replace the labour-intensive disaster site surveying that needs to timely investigate the disaster site quickly.

\section{ACKNOWLEDGEMENTS}

This research outputs are the part of the project "Development of forensic investigation for Disaster Scene", which is supported by the NDMI (National Disaster Management research Institute) under the project number NDMI-MA-2019-05-01. The authors would like to acknowledge the financial support of the NDMI.

\section{REFERENCES}

K. Eng, O., Strecha, C., Beyeler, A., Zufferey, JC, Floreano, D., Fua, P., Gervaix, F., 2011. The Accuracy of Automatic Photogrammetric Techniques on Ultra-Light Uav Imagery, International Archives of Photogrammetry, Remote Sensing and Spatial Information Sciences, Vol. XXXVIII-1 / C22, pp. 125130.

Kim, S.S., Song, B.G., Cho, S.B., Kim, H.J., 2019, Applicability of Drone Mapping for Natural Disaster Damage Investigation, Journal of Korean Society for Geospatial Information Science, Vol.27 No.2 March 2019 pp.13-21

Kim, S.S., Yoo, S.H., Park, J.S., Cho, S.B., Kim, T.H., 2018, Rapid Disaster Mapping through Data Integration from UAVs and Multi-sensors Mounted on Investigation Platforms of NDMI, Korea The International Archives of the Photogrammetry, Remote Sensing and Spatial Information Sciences, Volume XLII-3/W4, 2018 GeoInformation For Disaster Management (Gi4DM), 18-21 March 2018, Istanbul, Turkey. 
Mian, O., Lutes, J., Lipa, G., Hutton, J.J., Gavelle, E., Borghini, s., 2015. Direct Geo-referencing on Small Unmanned Aerial Platforms for Improved Reliability and Accuracy of Mapping Without the Need for Ground Control Points, International Archives of the Photogrammetry, Remote Sensing and Spatial Information Science, Vol. XL-1/W4, UAV-g 2015, York University, Toronto, Canada.

Ministry of the Interior and Safety, 2018, Guidelines for Planning Natural Disaster Investigation and Recovery Plans 2018.

Oh, Y. O, Choi, K. A. and Lee, I. P., 2017, Disaster Damage Detection Using Drone Aerial Images, In 2017 Joint Fall Conference proceedings of the Korean Society for Geo-spatial Information Science, pp. 213-214.

Pix4D, 2019, Accuracy of Pix4D Outputs, https://support.pix4d.com/hc/en-us/articles/202558889-

Accuracy-of-Pix4D-outputs (last date accessed at 05 March

Rehak, M., Mibillard, R., Skaloud, J. 2013. A Micro-UAV with the Capability of Direct Georeferencing, International Archives of the Photogrammetry, Remote Sensing and Spatial Information Science, Vol. XL-1/W2, UAV-g 2013, Rostock, Germany.

Turner, D., Lucieer, A., Wallace, L. 2013. Direct Georeferencing of Ultrahigh-Resolution UAV Imagery, IEEE Transaction on Geoscience and Remote Sensing, 52(5): 27382745.

Verhoenven, G. 2011, Taking Computer Vision AloftArchaelological Three-dimensional Reconstructions from Aerial Photographs with Photoscan, Archaeological Prospection. 18, 67-73. 